\title{
The solar physics FORWARD codes: Now with widgets!
}

\author{
Blake Forland $^{1,2} \dagger$, Sarah Gibson ${ }^{1}$, James Dove ${ }^{2}$ and Therese Kucera ${ }^{3}$ \\ ${ }^{1}$ High Altitude Observatory/National Center for Atmospheric Research \\ 3080 Center Green Dr. Boulder, CO, 80027, USA \\ email: lyon.bcf@gmail.com, sgibson@ucar.edu \\ ${ }^{2}$ Dept of Physics \& Metro State College Denver \\ NC 3123 Campus Box 691201 5th Street, Denver, CO, 80204-2005, USA \\ email: dove@msudenver.edu \\ ${ }^{3}$ NASA Goddard Space Flight Center \& Greenbelt, MD \\ 20771, USA \\ email: therese.a.kucera@nasa.gov
}

\begin{abstract}
We have developed a suite of forward-modeling IDL codes (FORWARD) to convert analytic models or simulation data cubes into coronal observables, allowing a direct comparison with observations. Observables such as extreme ultraviolet, soft X-ray, white light, and polarization images from the Coronal Multichannel Polarimeter (CoMP) can be reproduced. The observer's viewpoint is also incorporated in the FORWARD analysis and the codes can output the results in a variety of forms in order to easily create movies, Carrington maps, or simply observable information at a particular point in the plane of the sky. We present a newly developed front end to the FORWARD codes which utilizes IDL widgets to facilitate ease of use by the solar physics community. Our ultimate goal is to provide as useful a tool as possible for a broad range of scientific applications.
\end{abstract}

Keywords. Sun: prominences, Sun: corona, Sun: magnetic fields.

Solar coronal observables depend upon physical plasma properties such as density, temperature, velocity, and magnetic field, all of which are important constraints on theoretical models, but which in general are not themselves directly observable. Comparing models to data is not always straightforward, however, since each observable depends on coronal plasma properties in different ways. Moreover, the optically-thin corona results in observables that are integrated along the line of sight. A useful and general approach is forward modeling: taking a model-defined three-dimensional distribution of coronal plasma properties and performing line-of-sight integrals specific to a given coronal observable's dependence on these properties.

The "FORWARD" suite of forward-modeling IDL codes is designed to convert analytic models or simulation data cubes into coronal observables. It includes a set of analytic magnetohydrodynamic models and the capability to incorporate the output from any numerical MHD simulation or potential-field extrapolation, enabling modeling of magnetic structures ranging in size from that of active regions to the global corona. The output of these models can be translated into a broad range of observables, from white light to EUV to coronal Stokes polarimetry parameters in the infrared. These codes have broad applicability to coronal observations in general, and present a powerful tool for distinguishing between theoretical models through side-by-side comparison of their predictions to coronal data.

$\dagger$ NCAR is supported by the National Science Foundation 


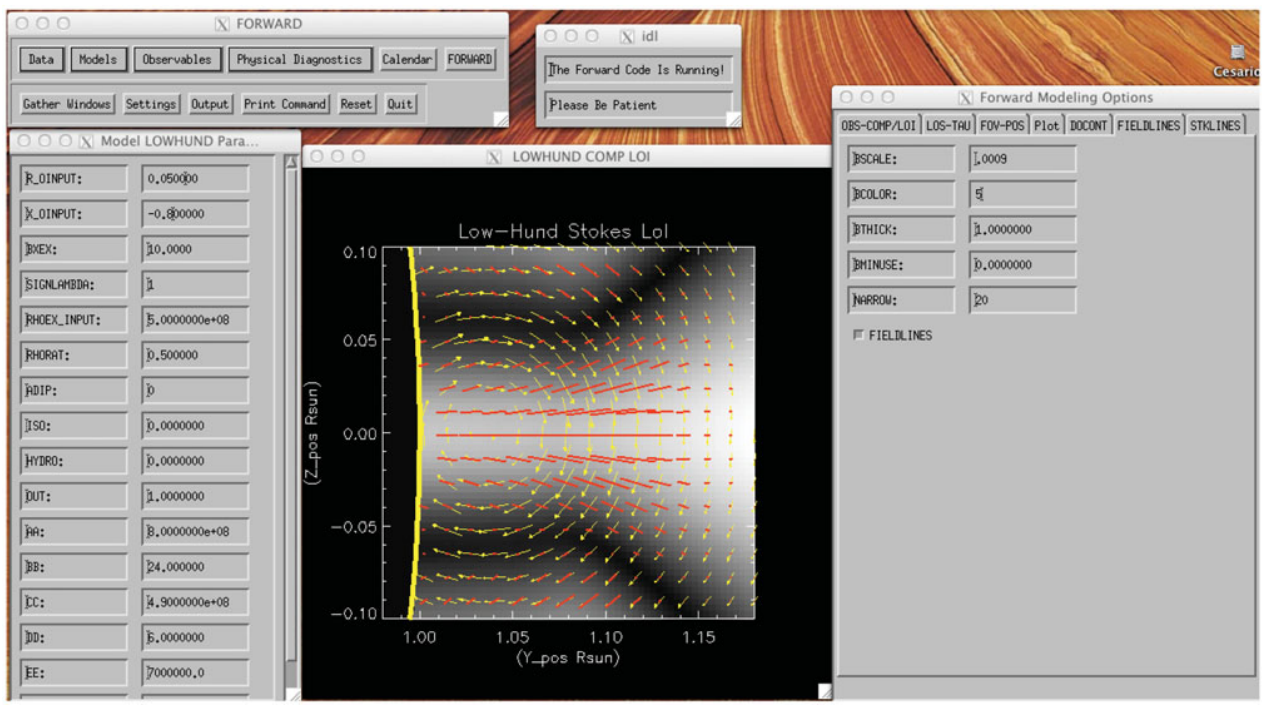

Figure 1. Example of widget interface of the FORWARD codes. Further information about how to access and install these codes available at http://people.hao.ucar.edu/sgibson/ FORWARD/

The FORWARD codes were recently used to extract the 3D morphology, density, and temperature of a coronal cavity from multiwavelength observations (Gibson et al. (2010), Schmit \& Gibson (2011), and Kucera et al. (2012)). They have also been used to analyze coronal magnometric properties in coronal cavities (Dove et al. (2011), Rachmeler et al. (2013), and Bạk-Stȩślicka et al. (2013)).

Recently, we have upgraded FORWARD to possess a widget interface (Figure 1). Our goal was to make this interface dynamic enough to adapt to parameter choice changes made by the user, but also to ongoing additions made to the base FORWARD codes. Structured default files for the models, observables, and associated parameters were set up within the FORWARD code so that the widget interface could automatically incorporate such changes. Overall, the goal was to allow users to apply the FORWARD codes to a broad range of scientific applications, without requiring a comprehensive understanding of its capabilities a priori.

The FORWARD codes are designed for ongoing expansion, with the ultimate goal of inclusion as a SolarSoft package. FORWARD enables model-data comparison at multiple wavelengths, enabling the user to effectively choose between models and ultimately reconstruct the three-dimensional coronal field. To this end, efforts are underway to expand FORWARD to incorporate radio and visible wavelength magnetometric diagnostics.

\section{References}

Bạk-Stȩślicka, U., Gibson, S. E., Fan, Y., Bethge, C., Forland, B., \& Rachmeler, L. A. 2013, Astrophys. J. in press; Arxiv 1304.7388

Dove, J., Gibson, S., Rachmeler, L. A., Tomczyk, S., \& Judge, P. 2011, Astrophys. J., 731, 1

Gibson, S. E., Kucera, T. A., et al. 2010, Astrophys. J., 723, 1133

Kucera, T. A., Gibson, S. E., Schmit, D. J., Landi, E., \& Tripathi, D. 2012, Astrophys. J., 757, 73

Rachmeler, L. A., Gibson, S. E., Dove, J. B., DeVore, C. R., \& Fan, Y. 2013, Solar Phys. in press, Arxiv 1304.7594

Schmit, D. J. \& Gibson, S. E. 2011, Astrophys. J., 733, 1 